\title{
Quality of Service Optimization based on HRM: A Case Study in Cameroonian Public Service
}

\author{
Abessolo Alo'o Ghislain \\ Department of Computer Science \\ University of Yaounde I
}

\author{
Atsa Etoundi Roger \\ Department of Computer Science \\ University of Yaounde I
}

\author{
Monthe Djiadeu Valery \\ Department of Computer Science \\ University of Yaounde I
}

\begin{abstract}
The search for a better quality of service in a changing environment requires governments to have more flexibility in the organization of work, a good grasp of emerging technologies and also a better consideration of human resources. The effectiveness of these resources will be even greater if they have the feeling of contributing in the search for solutions on new ways of producing services. Faced with economic, social and technological challenges, the quality of human resource management is a key success factor. The human resources management policy is often seen as less innovative and not dynamic; though many experts argue that improving quality of public service requires putting in place a stimulating human resource management, effective and motivating to state agents. Therefore, human resource management is seen as key with respect to competitiveness of administrations. The basis of optimal management of human resources depends on a good appointment policy to workposts. Indeed, governments in developing countries do not always produce the desired quality of service, for most human resources are not allocated according to their skill. While it is true that developing countries have embarked on extensive reform projects on the simplification of administrative procedures, it is especially important that a good policy be defined in order to assign skilled resources at work-posts in order to better perform those simplified procedures. However, it has been shown that the assignments and appointments, although discretionary in the public administration, are made arbitrarily and do not always permit to have the appropriated human resources at the appropriated places. Based on administrative business process modeling, this paper presents a protocol to ensure optimum assignment of human resources at work-posts in order to improve quality of service in public administration. For a given work-post, the protocol lists all human resources able to occupy this work-post by level of competence and experience in order to facilitate the choices of decision makers.
\end{abstract}

\section{Keywords}

Human Resource Management, Quality of Service Improvement, Competence and Experience Management, Administrative business process modeling, Human Resources Assignment, Work-posts Allocation, Human Resources Load Balancing

\section{INTRODUCTION}

Good public administration requires good human resources [7]. This has always been true, but its importance to the international community has probably never been more critical than it is today [7, 23]. Seldom, if ever, have the world's governments confronted a more daunting set of challenges that affect every nation, regardless of geographic location, political system, social structure or level of development [7]. Powerful forces, globalization, economic competition that cuts across national borders, social and political upheavals, technological change, threats of terrorism, and rapidly changing labor market place enormous burdens on governments [7,9]. The success of any government to respond effectively to these challenges is dictated largely by its ability to recruit and retain a talented workforce [21]. They also depend to the quality of the assignment of these human resources to the appropriated work-posts. Often overlooked since the advent of automation in public administration, the impact of the human entity on the functioning of administration seems indisputable today $[5,6]$. Their cognitive abilities shall for a long time make this, a key factor in the public administration [1]. However, the capabilities and behavior of the human resource are the basis of the introduction of new criteria for recognizing professional competence beyond only qualification required for a workpost [6]. Hence new assessment criteria appear. Autonomy, accountability and the relational aspect adds to traditional criteria of required skills (education, experience) and work complexity [20]. This change moves away from a classification of trades and positions to support more responsive work organizations, in response to the acceleration of change and differentiation in demand [24]. Improving the quality of service in a changing environment requires Public administrations to have more flexibility in the organization of work, better management of emerging technologies as well as a better consideration of its human resources. A new model seems to emerge: the competency model [17]. It relies on the commitment and enhancement of state employees and emphasizes on cooperation, autonomy, responsibility and allocation of work-post based on competency.

Today, the quality of service in the public sector in general and particularly in developing countries is based not only on simplification of procedures, but above all on the quality of human resources. The effectiveness of these resources will be even greater if they have the feeling of contributing in the search for solutions on new ways of producing services. Faced with economic, social and technological challenges, the quality of human resource management is a key success factor [6]. The human resources management policy is often seen as less innovative and not dynamic; though many experts argue that improving quality of public service requires putting in place a stimulating human resource management, effective and motivating to public officials. The Human resource management is therefore seen as one of the key points of competitiveness in administrations [2]. The effective use of their skills is essential to meeting the imposed performance targets and improving the quality of service rendered to the end users [5,6]. In addition, the basis of an optimal management of human resources depends on a good policy in the appointment of the latter to work-posts [16]. Indeed, public administrations in developing countries do not always 
produce the desired quality of service because human resources for most are not assigned according to their competency [3,7]. However, it has been shown that the assignments and appointments, although discretionary in public administrations, are made arbitrarily and do not always assign the required human resources at the right place [15]. As consequence, quality of service is negatively impacted thus rendering futile efforts to simplify procedures in the public sector. The allocation of a human resource to a work-post depends on the ability to carry out tasks associated to this work-post; this corresponds to the notion of competence $[5,20]$.

However, the notion of competence covers different concepts namely: competence in relation to a work, competence in relation to a task, competence levels and collective competence [5]. Some of these concepts have been studied in the literature, but generally unrelated to the assignments to work-posts $[5,8]$. Two aspects are identified in terms of the concept of competence of an agent to a work-post namely the qualification and experience [5,16]:

The qualification is the minimum required level of competence for the tasks associated with a workpost. It represents the required skill for minimum quality of service associated to a work-post in an administration. Therefore, a human resource assigned to a work-post can reach the minimum quality of service if he/she holds this qualification. Skilled human resources in relation to a work-post could not all carry out quickly the associated tasks: this is called experience of human resource.

Thus the qualification eliminates the possibility of assigning certain human resources that do not have the required skills to work-posts, the allocation of human resources influences the quality of service expected of these work-posts when the experiences of these human resources are different. On the other hand, human resources management problems depend on the time factor for which allocation decisions must be taken [7]. Typically, there are three levels of allocation decisions namely the strategic, tactical and operational level which corresponds to long-term, medium term and short term [21]. The objective of the short term is to schedule and allocate resources based on operational objectives (time and date of tasks, cost, etc.) [20]. At the operational level, human resource allocation approaches must incorporate a lot of constraints. First of all it is necessary to allocate to the various activities of a business process human resources with the required skills. This competence is clearly explained in business models where we distinguish the skills acquired and required [5]. However, it has little consideration in the literature and does not always include the collective dimension of human resource [18]. The quantitative constraint which defines the number of employees to be assigned to an activity is expressed in the formulation of Danzig on scheduling problem [22]. Human resource availability constraint incorporates many factors related to the socioeconomic environment of the entreprise such as vacation and legal limits of work. For this constraint class, the following problems are encountered in scheduling: day-off scheduling, shift scheduling and tour scheduling [22]. Finally, to these constraints is added the satisfaction of performance required by the enterprise's goals. Furthermore, several researches have shown that few studies have sought to incorporate the impact of skills on carrying out activities of an administrative process [9]. Some of them consider the human entity as a traditional resource which can be available or occupied or appointed to a work-post in an administration [21]. Others take into account the evolution of the skills of employees or groups of employees by modeling the evolution of their mastery of work $[6,8,20]$. All these works illustrate the diversity of human resource allocation problems that is due to the evolution of managerial practices, the multitude of application contexts, as well as differences in legislation between countries and sectors $[6,16]$.

This paper addresses the problem of human resources assignment in the public sector which has the distinction of having work-posts with job descriptions and occupied by quality human resources with the required profiles. The rest of the paper is organized as follows: section 2 presents the modeling of a public administration based on the work-posts and the required profiles; Section 3 gives an analysis of a well-trained public administration; Section 4 presents the protocol of optimal assignment of human resources at workposts and section 5 concluded and gives some perspectives.

\section{PUBLIC ADMINISTRATION MODELING}

Public administration is composed of a finite set of workposts for which human resources are assigned [3]. These human resources are involved in the execution of business processes to achieve a certain quality of service [8]. This quality of service will be maximal if and only if the human resources involved in the execution of business processes are competent and experienced; this implies that these human resources have been assigned to work-posts based on the required profiles and have more experience than others $[3,4]$. The quality of service will be minimal if and only if the human resources involved in the execution of business processes have minimal skills or qualifications but have no experience. This assumes that the allocations were made in accordance with the required profiles for different work-posts but human resources do not accumulate enough knowledge or experience in the execution of tasks at these work-posts. The quality of service will not be reached if and only if some resources involved in the execution of business processes do not have the required profiles for the work-posts to which they are assigned [3]. It appears in the latter situation that some resources are not properly assigned and that they will not achieve the expected quality of service at their work-posts. This situation is current in developing countries because the assignments are not always based on competency and when the expected quality of service is not reached, managers often undertake a reallocation of these human resources [3,7]. In this paper we shall model a public administration as a set of work-posts at which are assigned human resources for optimal performance of business processes.

\section{A. Administrative Process modelling}

This section gives the modelling of an administrative business process based on the definition of activity which is the key element in this model.

1) Activity Modelling: When dealing with the re-engineering of administrative workflows in order to meet stakeholders' needs, the main aspect to be taken into consideration is the activity concept [16]. The services delivered within an organization are obtained through the processing of a set of activities. In this write-up, it is conjectured that if an activity is well defined within an organization, it will be easy to carry out the analysis of its execution to attain a specific goal [6]. Therefore, the attributes defined in the following subsections are suitable for its efficient handling. 
Environment and execution context: Environment of the processing of activities within an administration is diverse. Therefore, it should be taken into consideration in the modelling of an administrative procedure to guarantee efficiency in the quality of services. It is assumed that the execution of a process will guarantee the required service if and only if this execution is operated in an environment that satisfies specific assumptions. Therefore, a procedure can be expected to operate correctly only when its environment operates correctly [1]. There is a great difference between the environment and the context concepts. In literature, some researchers use the two concepts in an interchangeable manner. In reality, as far as the processing of activities is concern within an organization, the environment describes the set of observable objects that are needed for the execution of a given activity [4]. However, the context describes the real state of these objects when the execution is taking place on the one hand and the condition from which the processing of the activity is taking place on the other hand. The environment $E_{X}$ within which the realization of an activity is supposed to take place is associated to a context of the realization within an administration [24]. This context is denoted by $C_{X}$. The context depends on the environment, meaning that if an activity is to be executed in two different environments, the associated execution context may also be different. So there is an unnoticeable correlation between an environment and the execution context of the activity.

Quality of service: Nowadays, the economy becomes more and more service-oriented. The service sector is growing not only due to the expanding public sector, but also because the industry becomes more service-oriented. To be able to survive and grow in the today's service oriented economy with global competition, an administration, an organization, or enterprise needs to pay close attention to the quality of services delivered to its service beneficiaries [19]. A definition of quality revolves around the idea that quality has to be judged on the assessment of the user or consumer of the service [9]. The construct of quality as conceptualized in the services literature is based on the perceived quality. Perceived quality is defined as the consumer's judgment about an entity's overall experience or superiority [17]. Perceived quality is also seen as a form of attitude, related to, but not the same as satisfaction, and resulting from a comparison of expectations with perceptions of performance [3]. The quality of service that should be obtained after the execution of a task and the whole business process will depend on the environment, the context of the execution and the resources associated in the execution activity. Based on this observation, the quality of service may vary between contexts, environments and associated human resource. Those external aspects of an activity may lead to an unacceptable quality of service. In order to capture the variability of the quality of service, this research proposes the quality of service to be specified as a quality of service interval noted by $Q o S_{A c t}$. $Q o S_{A c t}$ is formally defined by $\left[Q o S_{\min }, Q o S_{\max }\right]$ where $Q o S_{\min }$ and $Q o S_{\max }$ denote respectively the minimal and the maximal accepted quality of service delivered after the execution of an activity in a given work-post by a human resource. According to the quality of service $Q o S_{\text {exec }}$ obtained after the execution of an activity, the execution is said to be acceptable if and only if $Q o S_{\text {exec }} \leq Q o S_{\text {max }}$ and QoSexec $_{2} Q o S_{\text {min }}$ [3].

Execution Invariant: During the execution of an administrative procedure, the monitoring process is an important activity since erroneous situations can occur during the execution or some errors may be found after the execution. Therefore, it is possible that errors be founded after the execution of activities. When this happens, it negatively impacts on the quality of the service within the administration and also impacts the perception of the service by customers [13]. The constraints should be verified within the environment in order to trigger the execution and the end of the processing of the activity. Those constraints are the invariants of the activity and allow the control and the verification of its execution [11]. These invariants are not only focused on the inputs and outputs of the activity but also on the quality of service and the cost of service which is associated to the realization of the activity and to the availability of external resources that are required for its processing.

Execution constraint: In order to process activities within an administration, some constraints are required to be checked and satisfied. These constraints are applicable at two different levels, process level and activity level. Process level constraints specify which activities must be included within the process, and the flow dependencies within these activities including the control dependencies, such as sequence, alternative, parallel etc., and inter-activity temporal dependencies, such as relative deadlines. Activity level constraints constitute the specification of various properties of the individual activities within the process, including activity resources, such as applications, roles and performers, data, duration and deadline constraints. The constraints associated to the realization of the activity are the main factors that impact the quality of service. In administrative procedures, these entities are very important as the satisfaction of citizens is always relied on. The inability to satisfy the execution constraint will negatively impact the perception that the beneficiaries of the service have. There are three classes of constraints [12]: (1) the selection constraints that define what activities constitute the process, (2) the scheduling constraints that define when these activities are to be performed, both in terms of ordering as well as temporal dependencies, and lastly (3) the resource constraints that define which resources are required to perform the activities. Although, the various constraints are inter-related, it's still unusual to have model that take into consideration all these constraint abstractions.

2) Activity model: In order to better define procedures for their improvement within an administration, attributes defined above are required to be taken into consideration. The 
resulting model will be very important in decision making when dealing with quality of service management. For an efficient management of activities, an activity is modelled as a sigma algebra. Detail of the sigma algebra may be founded in [14].

Let $S=\{T K, Q o S, E I, E T C, R M, E C, E E, D x, R C$, $R S, S T, B G\}$ where:

TK denotes the name of the activity;

QoS denotes the set of quality of service that can be obtained after the execution of TK,

EI denotes the set execution invariants of $T K$,

ETC denotes the set of execution time constraints;

$E C$ denotes the set of execution contexts;

EE represents the set of execution environment;

$D x$ the set of required documents for the processing of $T K$;

$R S$ the required skills for the processing of $T K$;

$R M$ the required resources for the processing of

$T K$

$B G$ the cost for the execution of the activity;

ST the set of states that the environment may take based on the execution context of the activity TK.

Let $F$ denotes a set of functions defined in such a way that, iff $f$ is an element of $F$ then $f$ is in the form $f: S 1 \times S 2 \times \ldots \times S n \rightarrow S n+1$ with $S i \in S$.

Let I denotes the set of constraints that should be verified by activities of a given administrative business process, the different activities of a business process are represented by the set of functions denoted by F. Moreover, let S represents various resources that may be used for the execution of an activity.

An activity Act is defined by $A c t=(S, F, I)$. For each skill, a specific experience is needed in obtaining the projected quality of service. This skill and experience are linked to human resources. Based on the skill and the experience, the human resource in charge of the processing of the activity receives a payment related to the processing of the assigned activities. There is a strong relation between human resources and the budget of the administration. If the budget associated is not sufficient, the processing of the activity will not take place or will not end on time since some additional funds will be needed for the fulfilment of the activity. It should be noticed that in most developing countries, the wage bill represents $30 \%$ of the total budget, and failing to pay salaries increase the corruption in public administration.

The quality of service associated to the execution of an activity is influenced by the valuation of attributes defined above. By defining a specific valuation for a given activity, a specific activity called descriptive activity is defined. This means that a given formal definition of an activity is linked to a set of descriptive activities within an administration. For each concrete activity is associated a quality of service. In real life, two different descriptive activities may have the same quality of service. In this case, the descriptive activities with the same quality of service are said to be equivalent, and so are the associated valuation functions. Therefore, for a given activity and different valuations of attributes, the set of quality of services will have a minimum and a maximum quality of service. An activity associated with a valuation whose quality of service is minimum, is considered to be an initial algebra where the activity whose valuation gives the maximum quality of service is considered to be a terminal algebra. Some valuations will give the same quality of service after the realization of the activity, hence, a sigma congruence is defined among activities with the same signature in order to build a quotient algebra. For the rest of this paper, the concept of an activity is considered to be a class of equivalence of activities with the same quality of service.

Lemma 2.1: Let $T$ denote an activity to be undertaken, $E(T)$ a set of actions to be process during the execution of $T$ with $E(T) \subseteq S A P$, for each action $A c \in E(T), f(A c)$ denote the quality of service obtained after the processing of $A c$, the sum of the quality of service of the different actions gives the delivered quality of service of the associated activity i.e $Q_{o S}=\sum_{A c \in E(T)} f(A c)$.

Let $A_{T}$ denote the set of different executions of the activity $T$, based on the fact that different executions may lead to the same quality of service, a congruence cong is defined in $A_{T}$ in order to put together executions which deliver the same quality of service. The efficient management of the satisfaction of different stakeholders in the administration conducts the modelling process to define congruence in the $A_{Q o S}$ for a better control of the execution of various activities. In this work, the congruence is defined in such a way that only two classes of congruence are defined (a) the class dealing with accepted quality of service, and (b) the class with unaccepted quality of service.

Lemma 2.2: The execution of an activity $T$ will be said to be correct if and only if $Q o S_{T}$ is the class of accepted quality of service.

Lemma 2.3: Let $T$ be an activity, $T$ is said to be well formed if and only if the set of actions to be process is not empty i.e. $E(T) \neq \varnothing$, and $Q o S_{T}$ is the class of accepted quality of service.

Having defined the model of activity which constitutes the salient concept of a business process, the modelling of a business is done straightforward.

\section{2) Business process model}

A business process $B P$ is defined by $\left(S_{B P}, F_{B P}, E_{B P}\right)$ with $S_{B P}$ $=\left\{T K s, Q o S_{B P}, E I_{B P}, E T C_{B P}, R M_{B P}, E C_{B P}, E E_{B P}, D_{B P}, R C\right.$, $\left.R S_{B P}, S T_{B P}, B G_{B P}\right\}$ where $T K s$ denotes the set of task names and the remaining of sorts verify the following equations:
(E1) $\mathrm{QoS}_{\mathrm{BP}}=\sum_{t \in T K S} Q o S(t)$
(E2) $\mathrm{EI}_{\mathrm{BP}}=\bigcup_{t \in T K S} E I(t)$
(E3) $\mathrm{ETC}_{B P}=\cup_{t \in T K S} E T(t)$
(E4) $\mathrm{EE}_{\mathrm{BP}}=\bigcup_{t \in T K S} E E(t)$
(E5) $\mathrm{RM}_{B P}=\bigcup_{t \in T K S} R M(t)$
(E7) $\mathrm{D}_{\mathrm{BP}}=\cup_{t \in T K s} D(t)$
(E6) $\mathrm{EC}_{\mathrm{BP}}=\cup_{t \in T K S} E C(t)$
(E9) $\mathrm{ST}_{\mathrm{BP}}=\mathrm{U}_{t \in T K S} S T(t)$
(E8) $\mathrm{RS}_{\mathrm{BP}}=\mathrm{U}_{t \in T K S} R S(t)$
(E10) $\mathrm{BG}_{\mathrm{BP}}=\sum_{t \in T K S} B G(t)$

$F_{B P}$ defines the interface of the business process $B P$ in terms of different operations that can be process among sorts defined in $S_{\mathrm{BP}}$. Among these operations is for example the function that gives for each task the associated document for a given execution context, the follower function that gives for each task, the set of tasks that may be process just after its execution, the quality function that gives for each task and an execution context, the projected quality of service. $\mathrm{E}_{\boldsymbol{B} P}$ defines the set of different properties that are related to various operations and sorts.

In the public administration, business process defines the set of activities that are carried out within an administration in order to offer a specific service to the population. For the service to be accepted by the population, a certain quality of service should be defined. This quality of service is the aggregate of the partial quality of service associated to the activities. Each business process is seen as a Sigma-Algebra [14]. Moreover, to really understand the functioning of an 
administration, not only the structure of the business process should be understood but also the entire organisation of the administration must be mastered for a good quality of service management that will result to good decision making. The mastery of the public administration involves the modelling of different work posts within which human actors are appointed.

3) Work-post model: A work-post within an administration defines a position that a human actor may occupy in the administration. Each work-post has to deliver a certain quality of service. To ensure this requirement, the human resource must fulfil certain skills and experiences. For decision making, a work-post description should be defined. A work-post is denoted by the algebraic structure (S,F,E) with $\mathrm{S}=\{\mathrm{WP}, \mathrm{WSK}, \mathrm{SD}, \mathrm{QoS}, \mathrm{SAP}, \mathrm{TAP}\}$ denoting the set of attributes where WP is the name of the work-post; RSK is the required skills; SD is the services that may be delivered in this position; QoS the expected quality of service; SAP is the set of actions that can be performed in this position; TAP is the type of activities that are suitable to be performed. $\mathrm{F}$ denotes the set of relations among elements of $\mathrm{S}$, and $\mathrm{E}$ the set of properties that should be satisfied by the relations of $\mathrm{F}$ and even elements of $\mathrm{S}$. Among relations that can be defined between sorts of $\mathrm{S}$ are the mapping action that should be undertaken in order to produce a given service.

4) Human resource model: Tasks of a business process are mostly carried out by human resources within an administration. The human resource quality has then become a critical aspect to consider when dealing with the modelling of a business process. This even becomes a challenge when the quality of service and the reengineering of administrative procedure are considered. It has been well recognized that quality of a business process is influenced by the competency of the resources allocated to the process $[5,16]$. Still, most business process modelling techniques lack the capability to capture competence required to execute a function within a business process [5]. By doing so, some relevant attributes have to be taken into consideration. These attributes include [11,12]: suitability which is the capability to provide an appropriate function for specified user objectives; accuracy which refers to the capability of the function to provide the right or agreed results or effects with the needed degree of precision; security which relates to the capability of the function to protect information and data so that unauthorized resources cannot access them; reliability which is the capability of the function to maintain a specified level of performance when used under specified conditions; understandably which is the capability of the function to enable the resource to understand whether the function is suitable and how it can be used for particular functions and conditions of use; learnability which is the capability of the function to enable the user to learn it; time efficiency which is the capability of the function to provide appropriate response and processing times and throughput rates when performing its function, under stated conditions, resource utilisation which is the capability of the function to use appropriate amounts and types of resources under stated conditions; effectiveness which is the capability of the function to enable users to achieve specified goals with accuracy and completeness in a specified context of use; productivity which is the capability of the function to enable users to expend appropriate amounts of resources in relation to the effectiveness achieved in a specified context of use; safety is the capability of the function to achieve acceptable levels of risk of harm to people, process, property or the environment; user satisfaction which is the capability of the function to satisfy users in a specified context of use; robustness which is the degree to which a function can function correctly even in the presence of invalid, incomplete or conflicting inputs. Other attributes may be defined according the issue that is tackled.

In the government service, most activities are performed by human actors. Hence, the administration is organized in terms of work post. In each work post is an appointed a civil servant who deals with the processing of associated tasks. Each work post has a list of activities that can be performed and job description agenda for each task. It becomes difficult in most cases to appoint any civil servant to any work post as not all of them will have the same competences and experience to deal with the execution of any work post for a given work post. Before the appointment, skill and experience are therefore required for the civil servant to satisfy the projected quality of service in this post. This means that for each activity, the human actor in charge of its execution must have a certain competence and experience.

A human resource is denoted by the algebraic structure $(\mathrm{S}, \mathrm{F}, \mathrm{E})$ with $\mathrm{S}=\{\mathrm{HR}, \mathrm{RSK}, \mathrm{REX}, \mathrm{TAP}\}$ denoting the set of attributes where HR is the name of the human resource; RSK is the set of his skills; REX is the set of experiences which is the aggregated knowledge acquired in the past based on passed executions related to the activities; TAP is the type of activities that are suitable to be performed by HR. F denotes the set of relations among elements of $S$, and $E$ the set of properties that should be satisfied by the relations of $\mathrm{F}$ and even elements of S. Among relations that can be defined between sorts of $\mathrm{S}$ are the mapping action that should be undertaken in order to improve a given service.

5) Public administration model: A public administration is denoted by the algebraic structure (S,F,E) with $\mathrm{S}=\{\mathrm{PA}, \mathrm{WPs}$, HRs, BPs $\}$ denoting the set of attributes where PA is the name of the public administration; WPs is the set of the associated work-posts; HRs is the set of the human resources; BPs is the set of the associated business process. F denotes the set of relations among elements of $S$, and $E$ the set of properties that should be satisfied by the relations of $\mathrm{F}$ and even elements of S. Among relations that can be defined between sorts of $\mathrm{S}$ are the mapping action that give for each work-post, the human resource which is affected to this workpost.

Lemma 6.1 well defined administration: An administration is well-defined if and only if all their work-posts are occupied by qualified human resources i.e. with minimum required skills for the work-posts. This implies that the minimum quality of service will always be respected.

Lemma 6.2 Competent administration: An administration is said competent if and only if all work-posts are occupied by qualified and experienced human resources i.e. human resources have more experience in the processing of the associated tasks. This implies that the maximum quality of service is always guaranteed.

Lemma 6.3: incompetent administration: An administration is said to be incompetent if and only if the work-posts are occupied by certain human resources with no qualification compared with the work-posts they occupy. This implies that the quality of service expected will still not reached. In the following, we propose a protocol to ensure optimal allocation of resources within an administration. 


\section{THE HUMAN RESOURCES ASSIGMENT PROTOCOL}

Let $P A$ be an incompetent administration. The protocol defined for the optimal human resources assignment is given by the following steps:

Step1: Definition of required profiles for each work-post This is done from the definition of each work-post. Indeed, each work-post has a job description that gives all activities that can be performed and required profile or skills for each. This implies that the definition of a work-post brings out the required profile to be assigned at this post.

Step 2: Regroupment of human resources per competency Here we search the human resources that have the same skills in an organization. This consists to train skills classes for which human resources are associated.

\section{Notation:}

Let HRset $=\{H R 1, H R 2, \ldots, H R n\}:$ A set of human resources within an administration

FirstElement $(X)=$ Function which give the first element of the set $X$;

NberElement $(X)=$ function that returns the number of element of $X$;

SKILL $(H R)=$ Function that retourn the skills of the human resource $H R$;

$C L=$ Set of classes of human resource skills. This is a set of subsets of human resources grouped by type of skill.

Begin

$$
j=1
$$

$C L=\phi$

While $($ HRset $\neq \phi)$ do

CLj= FirstElement $($ HRset $)$

For $i=2$ to NberElement (HRset) do

If $((\operatorname{SKILL}(C L j)) \cap(\operatorname{SKILL}(H R i))=\operatorname{SKILL}(H R i))$ Then $C L j=C L j \cup\{H R i\}$

$$
\text { EndFor }
$$

HRset $=H R$ set $\backslash C L j$

$C L=C L \cup\{C L j\}$

$J=j+1$

EndWhile

End

\section{Step 3: Ranking of skill groups by experience level}

Here it involves reorganizing each class of skills in ascending order or in order of priority from the most experienced at the less experienced.

\section{Notation}

$C L=\{C L 1, C L 2, \ldots, C L n\}:$ Classes of skills within an administration.

CLi : Class of skill number $i$.

FirstSet $(C L)=$ function that returns the first set of the set $C L$.

$\operatorname{Exp}(H R)=$ Function that gives the experience of the human resource $H R$.

$\operatorname{Permute}(X, Y, Z)=$ Procedure who permute in the set $X$, the position of $Y$ to the position of $Z$.

\section{CE, Ej: Class of skills}

$$
\begin{aligned}
& \text { Begin } \\
& \mathrm{J}=1 \\
& \text { While }(\mathrm{CL} \neq \phi) \text { do } \\
& \quad \text { Ej }=\text { FirstSet }(C L) \\
& \quad C E=\text { FirstElement }(E j)
\end{aligned}
$$

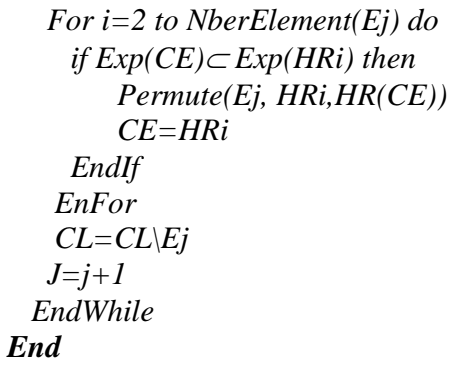

Step 4: The orientation of assignment to the work-posts according to the classes of skills

This is to find, for each work-post, the class of skill associated with it i.e. the class that contains the human resources with the required profiles. This provides orientations to policymakers about the quality of the resource to be assigned at a work-post.

\section{Notation}

$W P s e t=\{W P 1, W P 2, \ldots, W P n\}:$ A set of workposts within an administration.

$W P i=$ Work-post number $i$

$C L=$ classes of skill

Profil (WPi) is a function that takes as input a work-post and returns the minimum profile required at this post.

$C L i=$ class of skill number $i$

$P F i=$ variable $:$ classes of skills

$N b r e(X)$ is a function that returns the number of elements of the set $X$.

Affection $=$ the result containing couples work-post and class of skill. This is a set that gives for each work-post, the corresponding class of skills. For this, the decision maker has visibility with respect to human resources with the required skills in relation to a given work-post.

Nprofile $=$ the set which contains all work-posts for which there are no class of skills. For these work-posts, human resources have no required profiles.

NHR = non-qualified human resources i.e. human resources which have no required profile to be affected at the workposts.

$\mathrm{CL}=\{\mathrm{CL} 1, \mathrm{CL} 2 \ldots, \mathrm{CLn}\}$ : set of classes of skills defined above.

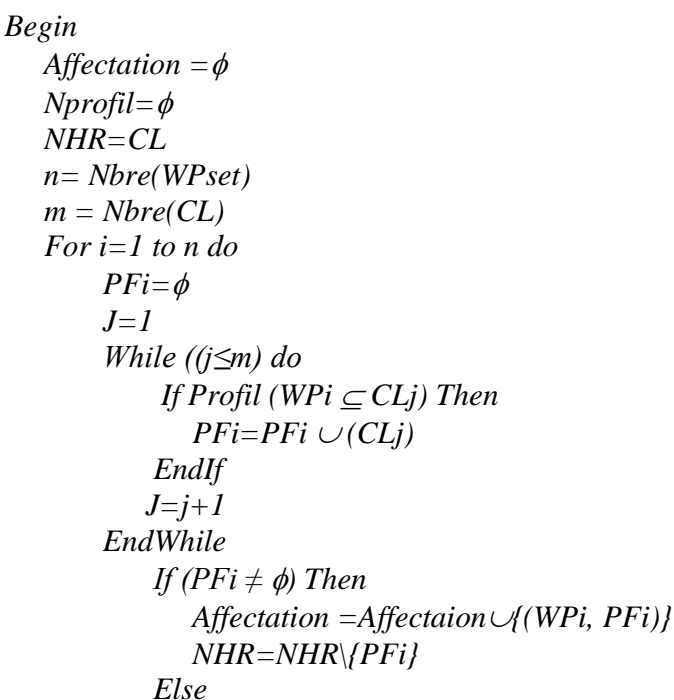




\author{
Nprofile $=$ Nprofile $\cup\{W P i\}$ \\ EnFor \\ EndIf \\ End
}

If $N H R$ is empty, then all human resources can be assigned at work-posts. Otherwise, we say that the human resources of $N R H$ have no required profile.

\section{A CASE STUDY IN THE \\ CAMEROONIAN PUBLIC SERVICE}

This section is not dealing with all the structures that are related to the management of human resource in the public service in Cameroon, but presents the information systems division. After presenting the division of the information systems and the status of assignments within this structure, we will apply the proposed protocol in order to highlight the optimal situation of assignments within the Division of Information Systems.

\section{A. Information Systems Division (ISD)}

The Information Systems Division has 14 work-posts, each with a clear profile that occupants must guarantee. In the public service, there are many documents who manage State personnel. For this study, we will use the work-post manual. Work-post manual is a document that allows a better understanding of the organization of the Cameroonian public service. This document describes, for each work-post, the required profile i.e. the set of minimum competencies that applicants should have. Concerning ISD, the following profiles are required:

Director of Information Systems (DIS): Master's degree in computer science or equivalent degree; 5 years of experience in administration; knowledge in IT project management; knowledge in administration of computer networks; knowledge in computer security; knowledge in Computer maintenance.

- Sub-director of Studies and Projects (SDSP): Master's degree in computer science or equivalent degree; 4 years of experience in administration; knowledge in IT project management; knowledge in development of IT applications.

- Sub-director of Exploitation, Infrastructure and Security (SDEIS): Master's degree in computer science or equivalent degree; 4 years of experience in administration; knowledge in administration of computer networks; knowledge in computer security; knowledge in Computer maintenance.

- Chief of service numberl at SDSP: BSc (Hons) in computer science or equivalent degree; 3 years of experience in administration; knowledge in IT project management; knowledge in development of IT applications.

- $\quad$ Chief of service number 2 at SDSP: BSc (Hons) in computer science or equivalent degree; 3 years of experience in administration; knowledge in IT project management; knowledge in development of IT applications

- Chief of service numberl at SDEIS: BSc (Hons) in computer science or equivalent degree; 3 years of experience in administration; knowledge in administration of computer networks; knowledge in computer security; knowledge in Computer maintenance.

- $\quad$ Chief of service number 2 at SDEIS: BSc (Hons) in computer science or equivalent degree; 3 years of experience in administration; knowledge in administration of computer networks; knowledge in computer security; knowledge in Computer maintenance.

- Agent in Information systems division: Bachelor's degree in computer sciences or equivalent degree; knowledge in IT project management; knowledge in administration of computer networks; knowledge in computer security; knowledge in Computer maintenance.

- In these work-posts are added the post of mailing agent who must have a ordinary level certificate, housekeeping agent who must have a first living certificate and a secretary who must have an Advanced level certificate and knowledge in secretariat duties.

The study allowed us to highlight the different work-posts with their occupants as follows:

Work-Post 1: Director of Information Systems (DIS):

Occupant name: A

Skills: \{Engineer of Post and Telecommunications; 25 years of experience in the Administration; Knowledge in management of telecommunications projects; Knowledge in administration of computer networks; Knowledge in Information Security; Knowledge in Computer Maintenance\}

Work-Post 2: Sub-director of Studies and Projects (SDSP)

Occupant name: B

Skills: \{Master's degree in computer science; 10 years of experience in the Administration; Knowledge in IT project management; Knowledge in development of IT applications; Knowledge in administration of computer networks; Knowledge in Information Security; Knowledge in computer Maintenance $\}$

Work-Post 3: Sub-director of Exploitation, Infrastructure and Security (SDEIS):

Occupant name: $\mathrm{C}$

Skills: Master's degree in computer science; 6 years of experience in the Administration; Knowledge in IT project management; Knowledge in development of IT applications; Knowledge in administration of computer networks; Knowledge in Information Security; Knowledge in computer Maintenance\}

Work-Post 4: Chief of service numberl at SDSP (CS1SDSP) Occupant name: D

Skills: \{Master's degree in computer science; 3 years of experience in the Administration; Knowledge in IT project management $\}$

Work-Post 5: Chief of service numberl at SDSP (CS2SDSP) Occupant name: E

Skills: \{Master's degree in computer science; 3 years of experience in the Administration; Knowledge in IT project management; Knowledge in development of IT applications; Knowledge in administration of computer networks; Knowledge in Information Security; Knowledge in computer Maintenance\}

Work-Post 6: Chief of service numberl at SDEIS (CS1SDEIS) Occupant name: $\mathrm{F}$

Skills: \{Bachelor's degree in computer sciences; 8 years of experience in the Administration; Knowledge in development of IT applications; Knowledge in administration of computer networks; Knowledge in Information Security; Knowledge in computer Maintenance\} 
Work-Post 7: Chief of service number 2 at SDEIS (CS2SDEIS)

Occupant name: $\mathrm{G}$

Skills: \{Bachelor's degree in computer sciences; 4 years of experience in the Administration; Knowledge in computer Maintenance

Work-Post 8: Agent number 1 at ISD (C1)

Occupant Name: $\mathrm{H}$

Skills: \{Master's degree in computer science; 4 years of experience in the Administration; Knowledge in IT project management; Knowledge in development of IT applications; Knowledge in administration of computer networks; Knowledge in Information Security; Knowledge in computer Maintenance\}

Work-Post 9: Agent number 2 at ISD (C2)

Occupant Name: I

Skills: \{Master's degree in computer science; 6 years of experience in the Administration; Knowledge in IT project management; Knowledge in development of IT applications; Knowledge in administration of computer networks; Knowledge in Information Security; Knowledge in computer Maintenance\}

Work-Post 10: Agent number 3 at ISD (C3)

Occupant name: $\mathrm{J}$

Skills: B Bachelor's degree in computer sciences; 6 years of experience in the Administration; Knowledge in Information Security; Knowledge in computer Maintenance \}

Work-Post 11: Agent number 4 at ISD (C4)

Occupant name: $\mathrm{K}$

Skills: \{Undergraduate diploma in computer sciences; 4 years of experience in the Administration; Knowledge in development of IT applications; Knowledge in computer Maintenance\}

Work-Post 12: Secretariat of ISD (SEC)

Occupant name: L

Skills: \{ordinary level certificate\}

Work-Post 13: Agent of Mail at ISD (ACL)

Occupant name: $\mathrm{M}$

Skills: \{advanced level certificate; Knowledge in secretariat duties \}

Work-Post 14: Office Assistant at ISD (OA)

Occupant Name: N

Skill: $\{$ advanced level certificate $\}$

B. Application of the proposed protocol in the Cameroonian public service

This section presents an application of the proposed protocol in the assignments of state employees in the division of the information systems in the Cameroonian public service.

Step 1: Definition of required profiles for each work-post. This is done from the definition of each work-post. Indeed, each work-post has a job description that gives all activities that can be performed and required profile or skills for each. This implies that the definition of a work-post brings out the required profile to be assigned at this post.

Step 2: Grouping human resources by competency. Here we search the human resources that have the same skills in an organization. This consists to train skills classes for which human resources are associated.

Let $H$ Rset $=\{A, B, C, D, E, F, G, H, I, J, K, L, M, N\}$ a set of human resources of DSI.
The skills of these resources are given as follows:

$\operatorname{SKILL}(A)=$ Engineer of Post and Telecommunications, knowledge in management of telecommunications projects, knowledge in administration of computer networks, knowledge in Information Security, knowledge in Computer Maintenance\}

SKILL $(B)=\{$ Master's degree in computer sciences, knowledge in IT project management, Knowledge in development of IT applications, knowledge in administration of computer networks, knowledge in Information Security, knowledge in

computer Maintenance

SKILL $(C)=\{$ Master's degree in computer sciences, knowledge in IT project management, Knowledge in development of IT applications, knowledge in administration

of computer networks, knowledge in Information Security, knowledge in computer Maintenance\}

$\operatorname{SKILL}(D)=\{$ Master's degree in computer sciences, knowledge in IT project management, knowledge in development of IT applications?

$S K I L L(E)=\{B S c($ Hons $)$ in computer sciences, knowledge in IT project management, Knowledge in development of IT applications, knowledge in administration of computer networks, knowledge in Information Security, knowledge in

computer Maintenance)

$\operatorname{SKILL}(F)=\{$ Bachelor's degree in computer sciences, knowledge in IT project management, Knowledge in development of IT applications, knowledge in administration of computer networks, knowledge in Information Security, knowledge in computer Maintenance\}

$\operatorname{SKILL}(G)=\{$ Bachelor's degree in computer sciences, knowledge in development of IT Applications, knowledge in administration of computer networks, Knowledge in Information Security, knowledge in computer Maintenance\}

SKILL $(H)=\{$ Master's degree in computer science, knowledge in IT project management, Knowledge in development of IT applications, knowledge in administration of computer networks, knowledge in Information Security, knowledge in computer Maintenance)

SKILL $(I)=\{$ Master's degree in computer sciences, knowledge in IT project management, Knowledge in development of IT applications, knowledge in administration

of computer networks, knowledge in Information Security, knowledge in computer Maintenance\}

SKILL $(J)=$ Bachelor's degree in computer sciences, knowledge in development of IT Applications, knowledge in administration of computer networks, Knowledge in Information Security, knowledge in computer Maintenance\}

$\operatorname{SKILL}(K)=$ Undergraduate diploma in computer sciences, knowledge in development of IT Applications, knowledge in computer Maintenance?

$\operatorname{SKILL}(L)=\{$ ordinary level certificate $\}$

$\operatorname{SKILL}(M)=\{$ Advanced level certificate, knowledge in secretariat duties\}

$\operatorname{SKILL}(N)=\{$ Advanced level certificate $\}$ 
The above definition of skills allows us to have the following classes of skills:

$C L 1=\{A\} ; C L 2=\{B, C, D, H, I\} ; C L 3=\{E\} ; C L 4=\{F, G, J\} ;$ $C L 5=\{K\} ; C L 6=\{L\} ;$

$C L 7=\{M\} ; C L 8=\{N\}$

Then, the set $\mathrm{CL}$ is given as follow: $C L=\{C L 1, C L 2, C L 3$, $C L 4, C L 5, C L 6, C L 7, C L 8\}$

Step 3: Classification of skill groups by experience level.

This classification concerns the classes $C L 2$ and CLA that have a number of human resources with the same skills. For this, the first action is to highlight the experiences of the associated human resources. This is done as follows:

$\operatorname{EXP}(B)=\{$ Agent at ISD (2 years), CS1 at ISD (3 years $)\}$

$\operatorname{EXP}(C)=\{$ Agent at ISD (3 years), CS2 at ISD (1 year), CS1 at ISD (4 years)\}

$\operatorname{EXP}(D)=\{$ Agent at ISD (2 years $)\} ; \operatorname{EXP}(H)=\{$ Agent at ISD (3 years)\};

$\operatorname{EXP}(I)=\{$ Agent at $I S D(4$ years $)\} ; \operatorname{EXP}(F)=\{$ Agent at $I S D(6$ years)\};

$\operatorname{EXP}(G)=\{$ Agent at $I S D(3$ years $)\} ; \operatorname{EXP}(J)=\{$ Agent at $I S D(5$ years)\}.

In view of the experiences of human resources involved, the following classification is done:

$C L 2=\{C, B, I, H, D\} ; C L 4=\{F, J, G\}$

Step 4: The orientation of assignment to the work-posts according to the classes of skills. This is to find, for each work-post, the class of skill associated with it i.e. the class that contains the human resources with the required profiles. This provides orientations to policymakers about the quality of the resources to be assigned at work-posts.

Let HRset and WPset respectively the set of human resources and the set of work-posts of the information systems division.

WPset $=\{D I S, \quad S D S P, \quad$ SDEIS, CSISDSP, CS2SDSP, CS1SDEIS, CS2SDEIS, C1, C2, C3, C4, ACL, SEC, OA); HRset $=\{A, B, C, D, E, F, G, H, I, J, K, L, M, N\}$

Let $C L$ the set of classes of skills previously defined. The execution of the protocol gives the following results:

$$
\begin{array}{ll}
-\quad & \text { Affectation }=\{(D I S, C L 2),(S D S P, C L 2),(S D E I S, \\
& C L 2),(C S 1 S D S P, C L 2, E),(C S 2 S D S P, C L 2, E), \\
& (C S 1 S D E I S, C L 2, E),(C S 2 S D E I S, C L 2, E),(C 1, \\
& C L 4),(C 2, C L 4),(C 4, C L 4),(S E C, M),(A C L, N), \\
& (O A, L)\} \\
-\quad & N R H=\{A, K\} \\
-\quad & \text { Nprofile }=\phi
\end{array}
$$

The above results reveal that human resources $A$ and $K$ have not required profiles to be assigned to the information systems division. Also, all posts should be occupied by the most experienced resources in relation to required profiles. Therefore, as the allocations are being made in this structure, the expected quality of service cannot be guaranteed because not only there are human resources assigned at work-posts for which they have no profile, but some of them are more experienced compared to other for their work-posts. Therefore, assignments or appointments here have not respected the required profiles at work-posts and this fact is observed at all levels in the Cameroonian public service.

\section{CONCLUSION}

In this paper, we presented an approach to make optimize the assignment of human resources at work-posts in public administration in order to improve the quality of service. To tackle this issue, the paper has first presented the problem faced by governments; particularly those of developing countries in terms of human resource assignment based on the profiles required at the work-posts. We realized that despite the vast field of simplification of administrative procedures to better meet the expectations of citizens by improving the quality of procedures, there is still a real problem because for a better quality of service, human resources should be assigned based on the required profiles at the work-posts. Appointments, although discretionary, still do not have the appropriated human resources at the appropriated work-posts and this has a negative impact on the quality of service. Policymakers are not sufficiently oriented in their choice because of lack of a mechanism which enables them to have visibility in terms of the performance of the human resources they manage.

In the second part of this work, we relied on the model of public administration to better define the human resource and work-post concepts. Thus, a work-post is seen as a set of activities to be performed in order to achieve a projected quality of service. For this, each work-post has a profile that gives guidance on the skill to be able to guaranty this expected quality of service. A human resource is defined based on the skills and experience gained in the implementation of activities of its competency. This implies that a good organization should consider these parameters to optimize the quality of service rendered to end users.

The next step of this write-up has consisted in the definition the protocol of optimal assignment of human resources at work-posts. For a given work-post, the protocol proposes human resources with the required skills. This set of human resources give a visibility according to the level of experience of each of them. Subsequently, we presented the results achieved following the implementation of the proposed protocol at the Information Systems Division in the Cameroonian Public Service. From these results, it appears that the Information Systems Division has a group of skilled and experienced human resources, but unfortunately that does not always hold the positions for which they are most competent and experienced. This negatively impacts the quality of service rendered. Discretion should be based on these guidelines to have an overall view of the quality of human resources in relation to the work-posts.

The problem solved here is encountered in most developing countries. We firmly believe that the proposed approach can be used in those countries to better address the human resource assignment problem which is also motivating because an employee assigned at a work-post to which he has skills and experience will be more motivated compared to another who is assigned to a position for which he is himself aware that he does not have the required skills.

However, we have not considered the issue of the allocation of tasks to the different structures of public administration. We realized that in developing countries, despite being assigned to a work-post for which one has the required skills and experience, quality of service is not always guaranteed because the hierarchy still assigns no tasks or activities to the appropriated structures. We believe as well that the work proposed here could be significantly improved if an orientation was given as to the tasks assigned to the structures. 


\section{ACKNOWLEDGMENTS}

The authors wish to thank the Ministry of the Public Service and Administrative Reform for its immense financial contribution. This work is being used to ameliorate the quality of assignment of human resources at work-posts.

\section{REFERENCES}

[1] Abadi, M., \& Plotkin, G. D. (1993). A Logical View of Composition. InTheoretical Computer Science...

[2] Amina Hameed and Shehla Amjad (2009), Impact of office design on employees' productivity: A case study of banking organization of abbottabad, pakistan. Journal of Public Affairs, Administration and Management, 3(1), 2009.

[3] Atsa Etoundi Roger and al (2010), Knowledge Management Driven Business Process andWorkflow Modeling within an

[4] AtsaEtoundi Roger (2011), "Atsero Method : AGuideline for Business Process and Workflow Modelling within an Entreprise". International Journal of Scientific Engineering Research

[5] Caetano, A., Pombinho, J., Tribolet, J. (2007): Representing organizational competencies. In: SAC, Proceedings 1257-1262

[6] Farouk Belkadi, Eric Bonjour, Maryvonne Dulmet. De la modélisation des situations de travail à la caractérisation des compétences : Une approche par logique floue. Journal Européen des Systèmes Automatiques (JESA), Lavoisier, 2009, 43 (1-2), pp.35-71.<10.3166/jesa.43.3571>. <hal-0043259>

[7] Government of Montenegro, (2011), Strategy of Public Administration Reform in Montenegro 2011-2016.

[8] Harzallah, M., Lecrere, M.: CommOnCV: Modelling the Competencies Underlying a Curriculum Vitae. SEKE, Proceedings. (2002)

[9] Hishamuddin Fitri Abu Hasan \& Azleen Ilias (2008), Service Quality and Student Satisfaction: A Case Study at Private Higher Education Institutions, International Business Research, Vol. 1, No. 3

[10] József Tick (2011), Application of P-graph-based workflow for administrative process modelling, Applied Machine Intelligence and Informatics (SAMI), 2011 IEEE 9th International Symposium

[11] Kuang-Hui Chiu (2006), Information flow over process. Journal of Information Optimisation Sciences,24(2):167192.
[12] Lu, R., Sadiq, S., Padmanabhan, V., \& Governatori, G. (2006). Using a temporal constraint network for business process execution. In Proceedings of the $17^{\text {th }}$ Australasian Database Conference-Volume 49 (pp. 157166). Australian Computer Society, Inc.

[13] Luciano Braseri \& Sam Guinea,(2005), Towards Dynamic Monitoring WS-BPEL Process, Lecture notes in Computer Science pp 269-282, Vol 3826

[14] Donald Sannella, Andrzej Tarlecki: Foundations of Algebraic Specification and Formal Software Development, Springer-Verlag Berlin Heidelberg, 14312654, 2012

[15] Reijers, H. A. \& Mendling, J. (2011), 'A study into the factors that influence the understandability of business process models', IEEE Transactions on Systems,Man, and Cybernetics-Part A: Systems and Humans 41(3), 449-462

[16] Neves, J., Caetano, A., Vasconcelos, A., Tribolet, J. (2001): Integrating knowledge into business processes. In: Proceedings of ISDEIS 2001

[17] Parasuraman, A., Zeithaml, V. A. \& Berry, L. L. (1990). Five imperatives for improving service quality. Sloan Management Review, 29-38.

[18] Reginald Lo, Service-based IT Cost Modeling and Identifying Cost Saving Opportunities, White paper.

[19] Stanka Preskar (2013): The Computer System Provides a Simpler Quality of Services in the Customs Procedure, Journal of Process Management - New Technologies, International Vol. 1, No.4

[20] Sandberg, J.: Understanding human competence at work: An interpretative approach. The Academy of Management Journal 43 (2000) 9-25

[21] Tanguy Lapegue. Planification de personnel avec affectation de taches : méthodes et application dans un contexte médical. Automatique. Ecole des Mines de Nantes, 2014, Français. 〈NNT : 2014EMNA0188>.

[22] Thompson, G. M. (2003). Labor Scheduling: A Commentary. Cornell Hospitality Quarterly, 44(56):149-155.

[23] Vuokko R, Mäkelä-Bengs P, Härkönen M. (2014), Improving health care service delivery with a national code service, Studies in Health Technology and Informatics.

[24] W.M.P. van der Aalst, F. Gottschalk1 M. Dumas, A.H.M. ter Hofstede, M. La Rosa, andJ. Mendling (2008), Correctness-preserving configuration of business process models. In Fundamental Approaches to Software Engineering (FASE 2008) 